\title{
AUTOMATIC HEADLIGHT DIMMER A PROTOTYPE FOR VEHICLES
}

\author{
Muralikrishnan. $\mathbf{R}^{1}$ \\ ${ }^{1}$ B.E, Electrical and Electronics Engineering, Sri Venkateswara College of Engineering, Tamil Nadu, India
}

\begin{abstract}
Headlights of vehicles pose a great danger during night driving. The drivers of most vehicles use high, bright beam while driving at night. This causes a discomfort to the person travelling from the opposite direction. He experiences a sudden glare for a short period of time. This is caused due to the high intense headlight beam from the other vehicle coming towards him from the opposite direction. We are expected to dim the headlight to avoid this glare. This glare causes a temporary blindness to a person resulting in road accidents during the night. To avoid such incidents, we have fabricated a prototype of automatic headlight dimmer. This automatically switches the high beam into low beam thus reducing the glare effect by sensing the approaching vehicle. It also eliminates the requirement of manual switching by the driver which is not done at all times. The construction, working and the advantages of this prototype model is discussed in detail in this paper.
\end{abstract}

Keywords: Headlight, automatic, dimmer, control, high beam, low beam, Kelvin (K).

\section{INTRODUCTION}

The requirement of headlight is very common during night travel. The same headlight which assists the driver for better vision during night travel is also responsible for many accidents that are being caused. The driver has the control of the headlight which can be switched from high beam (bright) to low beam (dim). The headlight has to be adjusted according to the light requirement by the driver. During pitch black conditions where there are no other sources of light, high beam is used to. On all other cases, low beam is preferred. But in a two-way traffic, there are vehicles plying on both sides of the road. So when the bright light from the headlight of a vehicle coming from the opposite direction falls on a person, it glares him for a certain amount of time. This causes disorientation to that driver. This discomfort will result in involuntary closing of the driver's eyes momentarily. This fraction of distraction is the prime cause of many road accidents. The prototype that is has been designed, reduces this problem by actually dimming down the bright headlight of our vehicle to low beam automatically when it senses a vehicle at close proximity approaching from the other direction. The entire working of the dimmer is a simple electronic circuitry arrangement which senses and switches the headlight according to the conditions required.

\section{CURRENT PROBLEM FACED BY MOTORISTS}

Motorists are facing a huge problem due to this high beam light which falls directly onto their eyes during driving. There are many medical facts and figures which support their problems of night driving.

\subsection{Troxler Effect}

In the medical world, Troxler effect is used to describe a kind of temporary blindness. It is otherwise known as the 'fading effect'. A study shows that if our eyes are exposed to a very bright light source of around 10,000 lumens, we experience a glare [1]. This glare is produced due to over exposure of the rods and cones inside our eye. Even after the source of glare is removed, an after-image remains in our eye that creates a blind spot. This phenomenon is called Troxler effect. This means that the driver's reaction time is increased by 1.4 seconds. For example, let us assume a motorist travelling at 60 miles per hour takes 0.5 seconds to react to a hazard and will stop within 41 feet. Due to Troxler effect, the same person travelling under the same conditions will take 0.9 seconds longer to react and hence will come to a complete halt only at 123 feet [2]. There is a huge difference of 82 feet. This is more than enough to cause a disaster on the road. This Troxler effect is across all ages. Any one exposed to sudden bright light experiences this Troxler effect.

\subsection{Accidents due to Troxler Effect}

As discussed earlier, there are many accidents caused due to Troxler effect. Many accident reports have been witnessed where a large vehicle, hitting a slow moving smaller vehicle while the latter is trying to over-take. Though it might be obvious to blame the driver, they claim to have not seen the smaller vehicle approaching. This is the most common example of illustrating the Troxler effect in our day-to-day life [3]. Due to excessive brightness, the driver of the large vehicle is blinded. So he is unable to notice the smaller vehicle even though it is right in front of him. This can be avoided if the headlight is dipped to low beam mode. According to Forbes, the statistics shown in Figure.1 gives the details of the 
accidents that had occurred in the year 2013 in Asia due to over-bright light. It shows clearly that India tops the list [4]. Hence, this becomes the major concern to think of a new innovative solution that is useful and also cost effective. This had led to the development of the automatic headlight dimmer prototype.

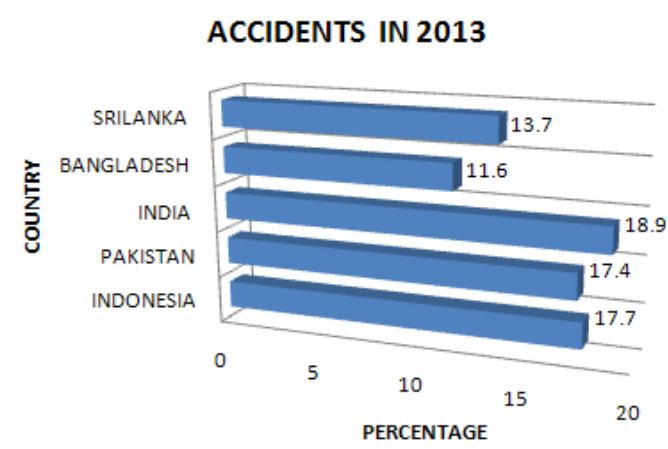

Fig-1: Accident report of Asia due to Troxler effect in 2013

\section{THE HEADLIGHT BEAMS}

The headlight of vehicles is fitted with two bulbs. One bulb is used for high beam and the other for the low beam. On an average, in India, the requirement of the headlight is essential from $6.00 \mathrm{pm}$ till $5.00 \mathrm{am}$. It is most essential during late night travels. The headlight can be switched between the bright and dip modes by the driver using a switch. The bright mode is used when there are no other sources of light on the streets to aid with driving. Long highways, a pitch black street with no lights are the ideal locations where one would use a bright beam [5]. The dip or the low beam is less intense than the bright beam. It is used under normal night driving conditions. The dip beam is aimed low at the road and gives less range. The high beam has a longer range but very less field coverage. Hence, dip beam is less intense (700 lumens) and high beam has a higher brightness index (1200 lumens) when tested under a standard distance of 50 feet from the vehicle [6]. Figure. 2 shows the range of the low beam and the high beam. The high beam since has a longer throw and a higher brightness index, will ultimately fall directly on the eyes of the driver coming on the other side of the traffic.

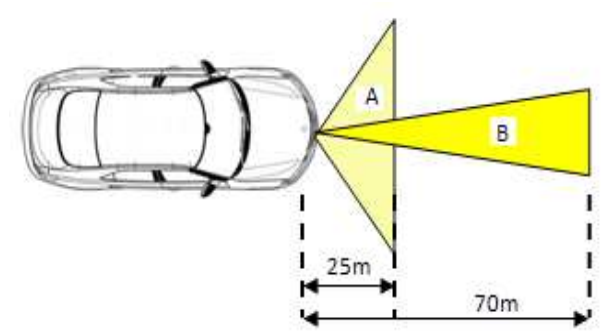

Fig-2: Range of low beam bulb (A) and high beam bulb (B) of a car
The angle of spread of the dip beam and the high beam is $135 \square$ and $15 \square$ respectively [5]. This again confirms on their range and spread. A human eye can withstand a brightness of around 1000 lumens when the source is at 20 feet [7]. Hence it is very important to make sure that our vehicle's bright (high) beam does not affect the driver coming from the opposite direction. As it is not possible to reduce the intensity of our headlight, all we have to do is to switch down to the dip beam until the traffic has passed away. This will ensure a safe and a friendly driving on the road during the night.

\section{THE PROTOTYPE CIRCUIT}

\subsection{The Layout}

The circuit is a simple assembly of commonly used circuit components. The layout is shown in Figure.3. The components have been chosen with utmost care and accuracy so as to keep the design simple and easy to implement.

\subsection{Components Used}

The various components used in the circuit are LDR (light dependent Resistor), two resistors as a potential divider, Transistor, Relay switch, LED bulbs and a Supply voltage.

The LDR is used to sense the incoming light. As the name suggests, its resistance value will vary according to the intensity of light that is incident upon its sensor. Higher the light intensity, lower will be its resistance.

The resistors used are a standard 0.25 watt, $1.6 \mathrm{kilo} \mathrm{ohm}$ and $30 \mathrm{ohm}$. They are used a potential divider in order to control the gate current to the transistor. To sense a wider range of light intensities, a POT can be used.

The transistor can be a BJT (bipolar junction transistor) or a MOSFET (metal oxide field effect transistor). If BJT is used, then the standard BC 547 is preferred. If a higher switching speed is required, then MOSFET - IRF 840 can be used.

The relay used is a $400 \mathrm{ohm}$ coil, 12 Volt, 5 terminal type. The normally-open contact is connected to the low beam bulb of the vehicle while the normally-closed contact is connected to the high beam bulb.

A supply of 12 volts is required for the circuit. It is taken from the vehicle's battery box. This is preferred for two reasons. First, it is a constant DC supply and second, there is no need for introducing a separate electrical supply source.

Two 0.25 watt LED bulbs are taken for simulating the headlights of the vehicle. One represents the bright mode bulb and the other, the low beam bulb. 


\subsection{Principle of Operation}

From the layout given if Figure.3, the basic idea about the working of the circuit can be understood. The LDR acts as a variable resistor. So the LDR, the two resistors form a potential divider network which will decide the current in the circuit. Thus, this balanced network gives a trigger to the gate/base of the transistor. The design of this particular circuit gets a trigger if there is a voltage imbalance in the circuit due to change in resistance of the LDR due to the light source.

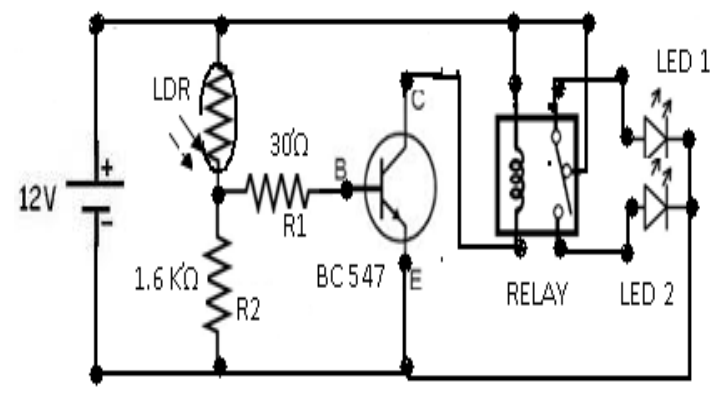

Fig-3: Schematic diagram of dimmer circuit

The basic operation is like that of a comparator. The transistor's output is connected to the relay coil. The bulbs are already connected to the relay contacts as mentioned earlier. LED 1 represents the high beam bulb which is in normally closed (NC) condition with the relay. LED 2 represents the low beam bulb of the vehicle which is at the normally open terminal (NO) of the relay. Whenever a high-intense light falls on the LDR, it's resistance drops thus creating an unbalance in the potential divider formed between the LDR, and two resistors R1 and R2. This will create a trigger current which turns on the transistor BC 547. The transistor gets into conduction mode and switches the relay. Hence the NC terminal will get disconnected and NO terminal will be switched. So, the vehicle's headlight which is in bright mode (LED 1) gets turned off and the low beam mode (LED 2) gets turned on by the relay. This happens when the vehicle from the opposite side crosses our vehicle. Thus as the other vehicle comes nearer, the intensity of that beam will increase and will hence switch our high beam light to low beam. As it moves away, the LDR will be turned away from the moving vehicle. So the LDR resistance increases and the bridge balances. There will hence be no trigger current and the relay switches back to its normal position. This will again turn on the bright beam mode bulb in our vehicle.

\section{THE ACTUAL CIRCUIT}

\subsection{Working of the Circuit}

Based on the prototype, an actual working model of the same circuit has been constructed. The exact same components have been used in its construction.
The source required is a $12 \mathrm{~V} \mathrm{DC}$ supply. We have taken the DC source from battery. But in real-time application, this can be substituted from the car's own battery pack. The headlights, LDR, transistor are all connected to the same DC supply (Figure.4).

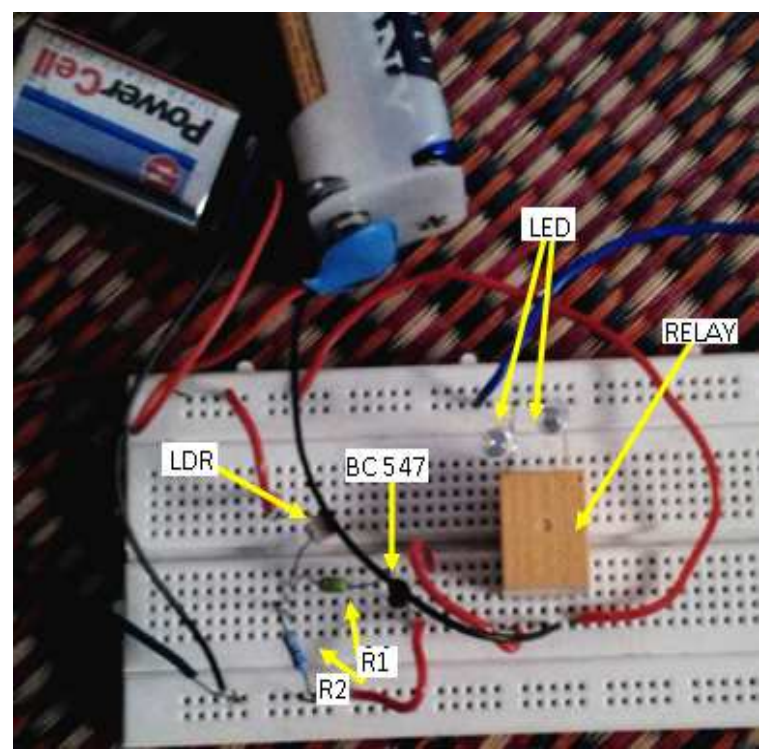

Fig-4: The Actual circuit

For its working, we need to simulate the condition where the LDR is exposed to a bright light, which is actually the headlight of another vehicle coming from the opposite side. Thus the LDR has a change in resistance.

Under normal conditions, the vehicle is using high beam bulb (shown as red LED in Figure.5.(a)). This case is considered under normal ideal conditions. At this stage, the relay is in NC condition.

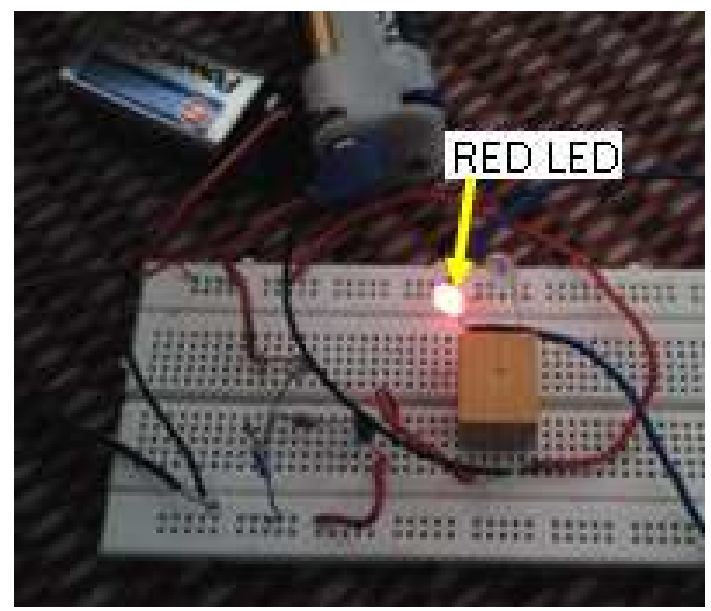

Fig-5(a): Normal driving conditions 
To understand the real-time working of this circuit, a high intense flash light has been used to simulate the event of an approaching vehicle. Whenever the LDR senses a light, it has to automatically switch from the high beam mode to the low beam mode. So, till the LDR senses the bright light (approaching vehicle), the bright bulb will be ON (RED LED). Once the intensity of the incident light goes beyond a particular value, it means that the vehicle is in close proximity of our vehicle. The LDR senses this threshold level and a drop in resistance is observed. This will send the transistor into conduction and the relay switches its contacts. Hence the NO contact which is connected to the low beam bulb gets turned $\mathrm{ON}$. As the relay is switched, the NC terminal is turned OFF. Thus the low beam light is switched ON automatically (shown by BLUE LED in Figure5(b)). The left side of the figure 5(b) is illuminated by a flash light to simulate the presence of an approaching vehicle.

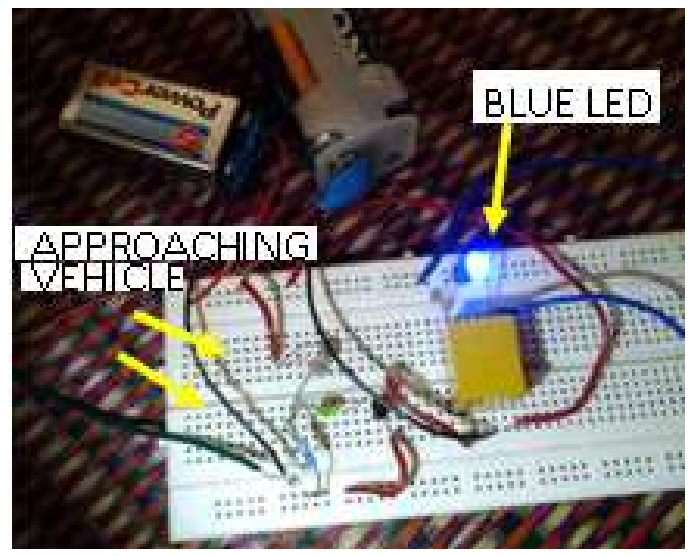

Fig-5(b): Automatic change over to low beam

\subsection{The Implementation}

The circuit had been constructed and proved to be working model. There are a few criteria which need to be addressed while placing this device in a real vehicle. They are:

- It should be kept at a safe place, protecting from external environment like rain, and dust.

- The placement of this circuit should be in line with the eye of the driver, so that it responds exactly in the same way how a driver would react to the bright light.

- The circuit should have a constant supply whenever the headlights are turned $\mathrm{ON}$.

- It should be compact and easy to install.

This device should be place in all the vehicles. By installing this device, each vehicle can independently operate on its own. Until the vehicle is encountered by an opposite vehicle, it can travel with high beam. Once it encounters an opposite vehicle, each of the two vehicles senses the opposite vehicle's light. Thus if either of the vehicles are using high beam, it switches to low beam. If the headlight is already in low beam, then no change occurs. As the vehicles cross each other, the intensity of light falling on the sensor decreases and the headlights switches back to their original mode.

There might be a question of other sources of light in the road like sign boards, street lights and buildings. But as LDR is used as the source and the placement of the device is highly directional, it is not affected by any of other light sources which might be present in vicinity. Moreover, the light from the vehicle's headlamp is of a distinct nature. The maximum spread angle of the headlight is $135 \square$ only [5]. The other sources will be located far away from the road and hence their spread angle will be very high. Hence by the time the spread light from other sources reach the sensor its intensity will be very much reduced below the triggering threshold level.

From the above discussions, it has been concluded that the device can be concealed in front of the car, near the wipers, at the base of the windscreen (Figure 6). The device is denoted as a red dot. This is the ideal place as it mimics the driver's line of sight and also safe from environmental factors and accidents.

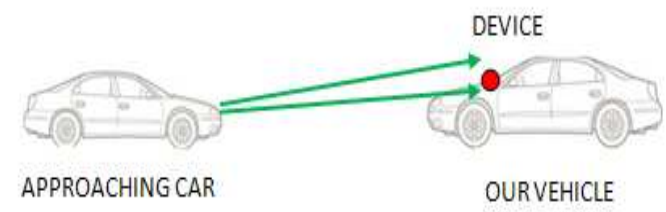

Fig-6: Positioning the device in vehicle

In the circuit, by using suitable adjustable resistors, we can tune the circuit's sensitivity to our requirement. It can be made sensitive for a wide range of light beam by just varying the balance condition of the potential divider network. Hence, the driver can manually adjust the sensitivity level so that it can be customized for his personal driving comfort.

\subsection{The Human Eye and its Sensitivity}

The human eye is a very sensitive organ. It works almost an entire day without any rest. Our eyes are adaptable for a particular range of vision. We have two visions namely the scotopic and photopic vision. Our eyes actually behave differently in different conditions. During bright surroundings, our eyes can resist up to $3 \mathrm{~cd} / \mathrm{m} 2$. This is the photopic vision. During dark and unlit conditions, our eye switches to scotopic vision which has a range of $30-45 \mu \mathrm{cd} / \mathrm{m} 2$. It takes 4 seconds for our eyes to change from photopic vision to scotopic vision. This is also an example of Troxler effect [8]. As the brightness increases, the strain to focus on an object increases. This will increase the response time of that person. Figure. 7 show that as the luminous index increases, the reflection percentage increases logarithmically. Higher the reflection index, lesser is the ability to perceive image in the eyes [9]. 


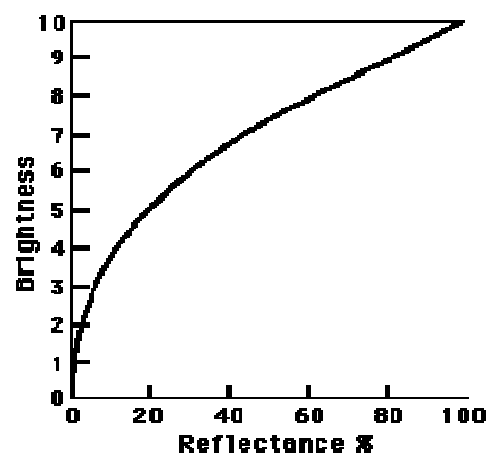

Fig-7: Relation between brightness and reflectance

Another study shown in Figure.8 represents the actual adaptable conditions and limits of the eye. This shows the variation of eye response to different luminosity and brightness [10]. The normal range, adaptable range and the glare limits are shown. Thus at the glare limit, the brightness and luminosity are maximum. Above the glare limit, the human eye is forced to close to avoid exposure to excessive light [11].

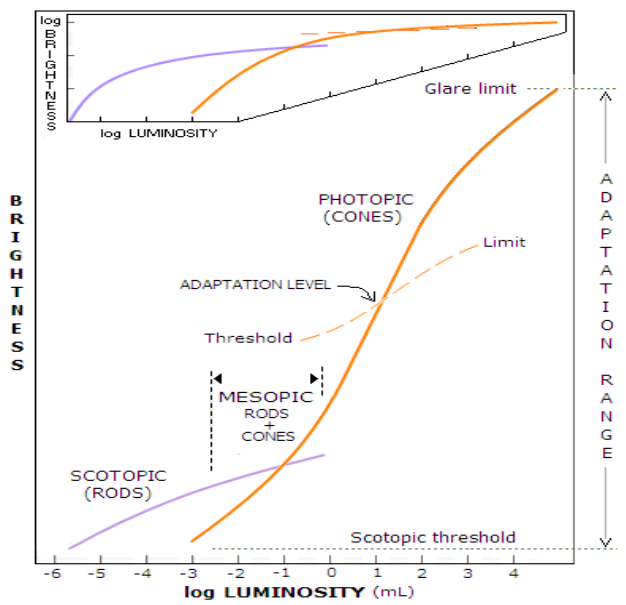

Fig-8: Eye response to various brightness levels

\section{CONCLUSIONS}

Glare during driving is a serious problem for drivers. This is caused due to the sudden exposure of our eyes to a very bright light; the bright headlights of vehicles in this case. This causes a temporary blindness called the Troxler effect. Eventually this becomes the major reason for night accidents. The driver should actually turn down the bright lights immediately to avoid glare to the other person which is not happening. Hence, is the idea for the design and development of a prototype circuit called the automatic headlight dimmer. It gives the driver to use high beam light when required. But it automatically switches the headlight to low beam when it senses a vehicle approaching from the opposite side. The circuit consists of simple and economical components which can be easily installed. The working and implementation of the prototype are discussed in detail. The effects of bright light on the human eye are also studied. Thus the implementation of this device in every vehicle in future will not only avoid accidents but also provide a safe and a comfortable driving.

\section{ACKNOWLEDGMENTS}

I thank Dr. M. Sivanantham, the management for helping and guiding me throughout this entire project. I am also grateful to the Chennai Traffic Police for providing me with the required facts and information for the statistics report.

\section{REFERENCES}

[1] C.Susana Martinez, S.L.Macknik and D.H.Hubel, The role of fixational eye movements in visual perception, Nature Reviews Neuroscience 5, 2004, pp.229-240.

[2] Ryota Kanai, Yukiyasu Kamitani and Universiteit Utrecht, Time-locked perceptual fading Induced by visual Transients, unpublished.

[3] S.Aishwarya, Bright Headlights a major cause of accidents, The Hindu, Online edition, May 02,2006.

[4] C.Guttman, High intensity headlights could cause road accidents by dazzling oncoming drivers, Eurotimes, April 2003.

[5] J.J.Fazzalaro, Limitations on Headlight brightness, OLD research report, Br.J.Ophthalmol. 87(1), pp.113$117,2003$.

[6] S.T.Chrysler, P.J.Carlson and H.Gene Hawkins, Imapcts of Retroreflectivity on sign Management, 01796-3, 2003.

[7] Lighting the future standard and high performance automotive halogen bulbs-Hella

[8] A.Majumder and S.Irani, Contrast Enhancement of Images using Human Contrast Sensitivity

[9] A.B.Watson, Temporal sensitivity, Vision RPS, vol.9, pp.947-952, 1969.

[10] R.Shapley, E.Kaplan and K.Purpura, Contrast sensitivity and light adaptation in photoreceptors or in the retinal network,1993.

[11] A.T.Bahill, Development, validation and sensitivity analyses of human eye movement models*, pp.311$357,1980$.

\section{BIOGRAPHIES}

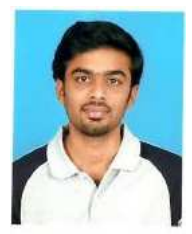

Muralikrishnan .R was born in Chennai, India on 24.12.1993 and completed his schooling from St.Michael's Academy in the year 2011. Currently pursuing the under graduate course, B.E (Electrical and Electronics Engineering) in Sri Venkateswara College Of Engineering, Sriperumbudur, India 
His areas of interests include nano-fabrications, Devices and circuit fabrication and embedded systems. $\mathrm{He}$ is an undergraduate engineering student. 